\title{
Solvable model for chimera states of coupled oscillators
}

\author{
Daniel M. Abrams, ${ }^{1}$ Renato E. Mirollo, ${ }^{2}$ Steven H. Strogatz, ${ }^{3, *}$ and Daniel A. Wiley ${ }^{4}$ \\ ${ }^{1}$ Department of Earth, Atmospheric, and Planetary Sciences, 54-621, \\ Massachusetts Institute of Technology, Cambridge, MA 02139, USA. \\ ${ }^{2}$ Department of Mathematics, Boston College, Chestnut Hill, MA 02467, USA. \\ ${ }^{3}$ Department of Theoretical and Applied Mechanics, Cornell University, Ithaca, NY 14853, USA. \\ ${ }^{4}$ Department of Mathematics, University of Maryland, College Park, MD 20742, USA.
}

(Dated: November 1, 2018)

\begin{abstract}
Networks of identical, symmetrically coupled oscillators can spontaneously split into synchronized and desynchronized sub-populations. Such chimera states were discovered in 2002, but are not well understood theoretically. Here we obtain the first exact results about the stability, dynamics, and bifurcations of chimera states by analyzing a minimal model consisting of two interacting populations of oscillators. Along with a completely synchronous state, the system displays stable chimeras, breathing chimeras, and saddle-node, Hopf and homoclinic bifurcations of chimeras.
\end{abstract}

PACS numbers: 05.45.Xt, 05.45.-a

Many creatures sleep with only half their brain at a time [1]. Such unihemispheric sleep was first reported in dolphins and other sea mammals, and has now been seen in birds and inferred in lizards [2]. When brain waves are recorded, the awake side of the brain shows desynchronized electrical activity, corresponding to millions of neurons oscillating out of phase, whereas the sleeping side is highly synchronized.

From a physicist's perspective, unihemispheric sleep suggests the following (admittedly, extremely idealized) problem: What's the simplest system of two oscillator populations, loosely analogous to the two hemispheres, such that one synchronizes while the other does not?

Our work in this direction was motivated by a series of recent findings in nonlinear dynamics $3,4,4,5$, 6, 7, 8]. In 2002, Kuramoto and Battogtokh reported that arrays of nonlocally coupled oscillators could spontaneously split into synchronized and desynchronized subpopulations [3]. The existence of such "chimera states" came as a surprise, given that the oscillators were identical and symmetrically coupled. On a one-dimensional ring $\underline{3}$, 44 the chimera took the form of synchronized domain next to a desynchronized one. In two dimensions, it appeared as a strange new kind of spiral wave 5, with phase-locked oscillators in its arms coexisting with phaserandomized oscillators in its core - a circumstance made possible only by the nonlocality of the coupling. These phenomena were unprecedented in studies of pattern formation [9] and synchronization [10] in physics, chemistry, and biology, and remain poorly understood.

Previous mathematical studies of chimera states have assumed that they are statistically stationary $3,3,4,5,6$, 7]. What has been lacking is an analysis of their dynamics, stability, and bifurcations.

In this Letter we obtain the first such results by considering the simplest model that supports chimera states: a pair of oscillator populations in which each oscillator is coupled equally to all the others in its group, and less strongly to those in the other group. For this model we solve for the stationary chimeras and delineate where they exist in parameter space. An unexpected finding is that chimeras need not be stationary. They can breathe. Then the phase coherence in the desynchronized population waxes and wanes, while the phase difference between the two populations begins to wobble.

The governing equations for the model are

$$
\frac{d \theta_{i}^{\sigma}}{d t}=\omega+\sum_{\sigma^{\prime}=1}^{2} \frac{K_{\sigma \sigma^{\prime}}}{N_{\sigma^{\prime}}} \sum_{j=1}^{N_{\sigma^{\prime}}} \sin \left(\theta_{j}^{\sigma^{\prime}}-\theta_{i}^{\sigma}-\alpha\right)
$$

where $\sigma=1,2$ and $N_{\sigma}$ is the number of oscillators in population $\sigma$. The oscillators are assumed identical, so the frequency $\omega$ and phase lag $\alpha$ are the same for all of them. The strength of the coupling from oscillators in $\sigma^{\prime}$ onto those in $\sigma$ is given by $K_{\sigma \sigma^{\prime}}$. To facilitate comparison with earlier work, we suppose that $K_{11}=K_{22}=\mu>0$, and $K_{12}=K_{21}=\nu>0$, with $\mu>\nu$. Thus, the coupling within a group is stronger than the coupling between groups. This corresponds to the assumption [3, 4, [5] of a nonlocal coupling that decreases with distance. By rescaling time, we may set $\mu+\nu=1$. It also proves useful to define the parameters $A=\mu-\nu$ and $\beta=\pi / 2-$ $\alpha$, because, as we'll show, chimeras exist only if these quantities are small enough.

Simulations of Eq. (1) display two types of behavior. For many initial conditions, the system approaches the synchronized state where all $\theta$ 's are equal. Otherwise it evolves to a chimera state (Fig. 11). The oscillators in group 1 are in sync; those in group 2 are not.

Figure 2 illustrates the dynamics of chimera states. We plot the phase coherence of the desynchronized population, as quantified by the order parameter $r(t)=$ $\left|\left\langle e^{i \theta_{j}(t)}\right\rangle_{2}\right|$, where the angle brackets denote an average over all oscillators in population $\sigma=2$. In Fig. 2(a) the order parameter remains constant, except for slight fluctuations due to finite-size effects. Thus, this chimera 


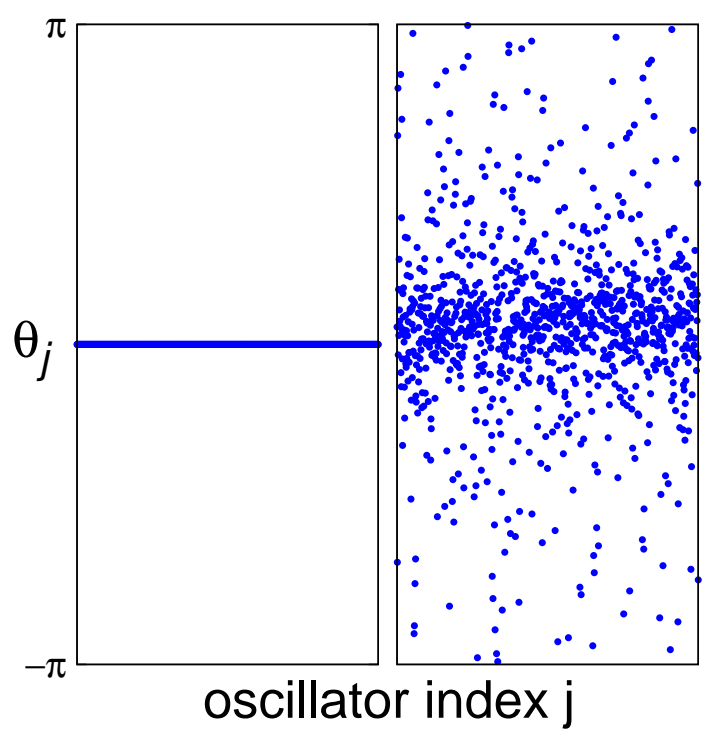

FIG. 1: Snapshot of a chimera state, obtained by numerical integration of (11) with $\beta=0.1, A=0.2$, and $N_{1}=N_{2}=$ 1024. The two populations are shown side by side.

is stable and statistically stationary. However, if we increase $\mu$ (the coupling within a population) relative to $\nu$ (the coupling between populations), the stationary state can lose stability. Now the order parameter pulsates, and the chimera starts to breathe (Fig. 2(b)). The breathing cycle lengthens as we increase the disparity $A=\mu-\nu$ between the couplings (Fig. 2(c)). At a critical disparity, the breathing period becomes infinite. Beyond that, the chimera disappears and the synchronized state becomes a global attractor.

To explain these results, we analyze Eq. (1) in the continuum limit where $N_{\sigma} \rightarrow \infty$ for $\sigma=1,2$. Then Eq. (1) gives rise to the continuity equations

$$
\frac{\partial f^{\sigma}}{\partial t}+\frac{\partial}{\partial \theta}\left(f^{\sigma} v^{\sigma}\right)=0
$$

where $f^{\sigma}(\theta, t)$ is the probability density of oscillators in population $\sigma$, and $v^{\sigma}(\theta, t)$ is their velocity, given by

$$
v^{\sigma}(\theta, t)=\omega+\sum_{\sigma^{\prime}=1}^{2} K_{\sigma \sigma^{\prime}} \int \sin \left(\theta^{\prime}-\theta-\alpha\right) f^{\sigma^{\prime}}\left(\theta^{\prime}, t\right) d \theta^{\prime} .
$$

(Note that we dropped the superscripts on $\theta$ to ease the notation. Thus, $\theta$ means $\theta^{\sigma}$ and $\theta^{\prime}$ means $\theta^{\sigma^{\prime}}$.) If we define a complex order parameter

$$
z_{\sigma}(t)=\sum_{\sigma^{\prime}=1}^{2} K_{\sigma \sigma^{\prime}} \int e^{i \theta^{\prime}} f^{\sigma^{\prime}}\left(\theta^{\prime}, t\right) d \theta^{\prime},
$$

then $v^{\sigma}(\theta)$ simplifies to

$$
\begin{aligned}
v^{\sigma}(\theta, t) & =\omega+\operatorname{Im}\left[e^{-i \theta} e^{-i \alpha} z_{\sigma}(t)\right] \\
& =\omega+\frac{1}{2 i}\left(z_{\sigma} e^{-i \alpha} e^{-i \theta}-z_{\sigma}^{*} e^{i \alpha} e^{i \theta}\right),
\end{aligned}
$$

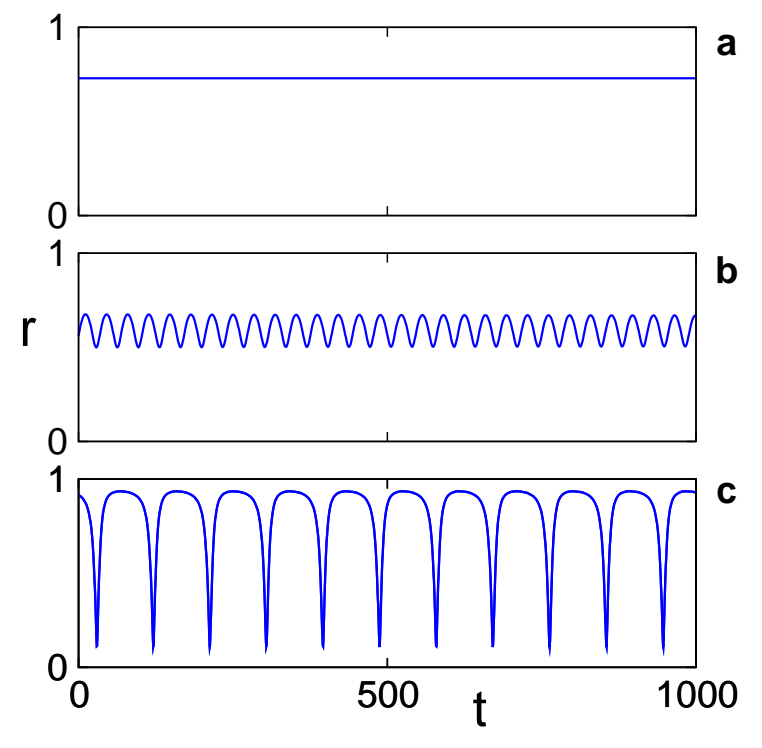

FIG. 2: Order parameter $r$ versus time. In all three panels, $N_{1}=N_{2}=128$ and $\beta=0.1$. (a) $A=0.2$ : stable chimera; (b) $A=0.28$ : breathing chimera; (c) $A=0.35$ : long-period breather. Numerical integration began from an initial condition close to the chimera state, and plots shown begin after allowing a transient time of 2000 units.

where the $*$ denotes complex conjugate.

Following Ott and Antonsen [11], we now consider a special class of density functions $f^{\sigma}$ that have the form of a Poisson kernel. The remarkable fact that Ott and Antonsen discovered is that such kernels satisfy the governing equations exactly, if a certain low-dimensional system of ODEs is satisfied. In other words, for this family of densities, the dynamics reduce from infinite dimensional to finite (and low) dimensional. (Numerical evidence suggests that all attractors lie in this family, but proving this remains an open problem.)

Specifically, let

$$
f^{\sigma}(\theta, t)=\frac{1}{2 \pi}\left\{1+\left[\sum_{n=1}^{\infty}\left(a_{\sigma}(t) e^{i \theta}\right)^{n}+c . c .\right]\right\} .
$$

What's special here is that we use the same function $a_{\sigma}(t)$ in all the Fourier harmonics, except that $a_{\sigma}$ is raised to the $n^{\text {th }}$ power in the $n^{\text {th }}$ harmonic. Inserting this $f^{\sigma}$ into the governing equations, one finds that this is an exact solution, as long as

$$
\dot{a}_{\sigma}+i \omega a_{\sigma}+\frac{1}{2}\left[a_{\sigma}^{2} z_{\sigma} e^{-i \alpha}-z_{\sigma}^{*} e^{i \alpha}\right]=0 .
$$

Instead of infinitely many amplitude equations, we have just one. (It's the same equation for all $n$.)

To close the system, we express the complex order parameter $z_{\sigma}$ in terms of $a_{\sigma}$. Inserting the Poisson ker- 
nel (6) into Eq. (4), and performing the integrals, yields

$$
z_{\sigma}(t)=\sum_{\sigma^{\prime}=1}^{2} K_{\sigma \sigma^{\prime}} a_{\sigma^{\prime}}^{*}(t)
$$

by orthogonality. Thus the amplitude equations become

$$
\begin{aligned}
0= & \dot{a}_{1}+i \omega a_{1}+\frac{1}{2} a_{1}^{2}\left(K_{11} a_{1}^{*}+K_{12} a_{2}^{*}\right) e^{-i \alpha} \\
& -\frac{1}{2}\left(K_{11} a_{1}+K_{12} a_{2}\right) e^{i \alpha}
\end{aligned}
$$

and similarly for $\dot{a}_{2}$, with 1's and 2's interchanged.

Rewrite the amplitude equations using polar coordinates $\rho$ and $\phi$, defined by $a_{\sigma}=\rho_{\sigma} e^{-i \phi_{\sigma}}, \sigma=1,2$. (The negative sign is included in the definition of $\phi$ so that the Poisson kernel $f^{\sigma}$ converges to $\delta\left(\theta-\phi_{\sigma}\right)$, not $\delta\left(\theta+\phi_{\sigma}\right)$, as $\rho \rightarrow 1$ from below. Thus $\phi_{\sigma}$ can be interpreted as the "center" of the density $f^{\sigma}$, and $\rho_{\sigma}$ measures how sharply peaked it is.) Then Eq. (9) becomes

$$
\begin{aligned}
0= & \dot{\rho}_{1}+\frac{\rho_{1}^{2}-1}{2}\left[\mu \rho_{1} \cos \alpha+\nu \rho_{2} \cos \left(\phi_{2}-\phi_{1}-\alpha\right)\right] \\
0= & -\rho_{1} \dot{\phi}_{1}+\rho_{1} \omega \\
& -\frac{1+\rho_{1}^{2}}{2}\left[\mu \rho_{1} \sin \alpha+\nu \rho_{2} \sin \left(\phi_{1}-\phi_{2}+\alpha\right)\right]
\end{aligned}
$$

and similarly for $\dot{\rho}_{2}$ and $\dot{\phi}_{2}$, with 1's and 2's interchanged.

Now consider the case of chimera states, for which one population is in sync while the other is not. Taking $\sigma=1$ to be the synchronized population, we set $\rho_{1} \equiv 1$, corresponding to a $\delta$-function for that group. Note that $\rho_{1} \equiv 1$ satisfies the governing equations for all time, since $\dot{\rho}_{1}=0$ when $\rho_{1}=1$. Hence the condition $\rho_{1} \equiv 1$ defines an invariant manifold, on which the dynamics reduce to

$$
\begin{aligned}
\dot{\phi}_{1} & =\omega-\mu \sin \alpha-\nu r \sin (\psi+\alpha) \\
\dot{r} & =\frac{1-r^{2}}{2}[\mu r \cos \alpha+\nu \cos (\psi-\alpha)] \\
\dot{\phi}_{2} & =\omega-\frac{1+r^{2}}{2 r}[\mu r \sin \alpha+\nu \sin (\alpha-\psi)]
\end{aligned}
$$

where we've defined $r=\rho_{2}$ and $\psi=\phi_{1}-\phi_{2}$. The $(r, \psi)$ dynamics decouple, yielding a $2-D$ system given by

$$
\begin{aligned}
\dot{r}= & \frac{1-r^{2}}{2}[\mu r \cos \alpha+\nu \cos (\psi-\alpha)] \\
\dot{\psi}= & \frac{1+r^{2}}{2 r}[\mu r \sin \alpha-\nu \sin (\psi-\alpha)]-\mu \sin \alpha \\
& -\nu r \sin (\psi+\alpha) .
\end{aligned}
$$

This system has a trivial fixed point $r=1, \psi=0$ (perfectly synchronized state) which always exists. The non-trivial fixed points correspond to stationary chimera states, in which the local order parameters $\rho_{1}(t) \equiv 1$ and $\rho_{2}(t)=r(t)$ remain constant, as does the phase difference $\psi(t)=\phi_{1}(t)-\phi_{2}(t)$, despite the fact that the individual

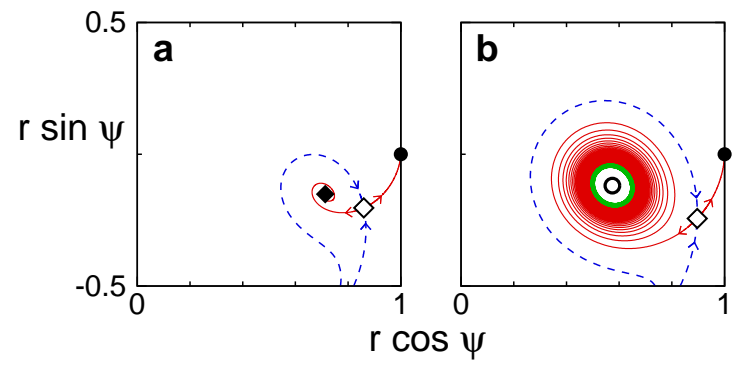

FIG. 3: Phase portraits for Eq. (12), regarding $r$ and $\psi$ as polar coordinates. Parameters as in Figs. 2(a),(b), respectively. (a) Stable chimera (solid diamond). (b) Breathing chimera, shown as a stable limit cycle (thick curve) about unstable chimera (open dot). In both panels: open diamond, saddle chimera; thin solid line, unstable manifold; dashed line, stable manifold; solid dot, stable synchronized state.

microscopic oscillators in population $\sigma=2$ continue to move in a desynchronized fashion.

Figure 3 plots typical phase portraits for (12). Figure B(a) shows a stable chimera state coexisting with the stable synchronized state; the basin boundary between them is defined by the stable manifold of a saddle chimera. As we increase the disparity $A$ between the couplings $\mu$ and $\nu$, the chimera becomes less stable and eventually undergoes a supercritical Hopf bifurcation, creating a stable limit cycle (Fig. 3(b)), the counterpart of the breathing chimera of Fig. 2(b).

Additional phase plane analysis (not shown) reveals two other bifurcations. With further increases in $A$, the limit cycle expands and approaches the saddle. Meanwhile, its period lengthens, which accounts for the behavior seen earlier in Fig. 2(c). At sufficiently large $A$ the cycle touches the saddle point and destroys itself in a homoclinic bifurcation. On the other hand, if $A$ is decreased from its value in Fig. 3(a), the stable and saddle chimeras in Fig. 3(a) approach each other, and eventually coalesce and annihilate in a saddle-node bifurcation.

Figure 4 summarizes the bifurcations and stability regions for the system. In the rest of the paper we outline the analysis leading to these results.

To calculate the fixed points for Eq. (12), we set $\dot{r}=0$ and $r \neq 1$ (since group $\sigma=2$ is desynchronized) and obtain $\mu r \cos \alpha+\nu \cos (\alpha-\psi)=0$. Substituting $\mu=$ $(1+A) / 2, \nu=(1-A) / 2$ and $\beta=\pi / 2-\alpha$ into $\dot{r}=0$ and solving for $A$ yields

$$
A=\frac{\sin (\beta+\psi)+r \sin \beta}{\sin (\beta+\psi)-r \sin \beta}
$$

at a fixed point. Then imposing $\dot{\psi}=0$ and using the expression for $A$ above, we find

$$
r=\sqrt{\frac{\sin (2 \beta+\psi)}{\sin (2 \beta-\psi)+2 \sin \psi}} .
$$




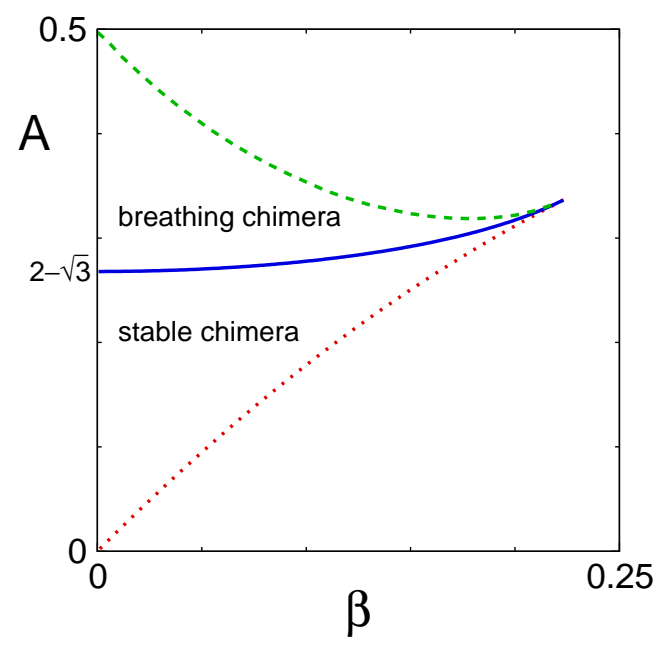

FIG. 4: Stability diagram for chimera states. Bifurcation curves: saddle-node (dotted) and supercritical Hopf (solid), both found analytically; homoclinic (dashed), found numerically. All three curves intersect at a Takens-Bogdanov point $(\beta, A)=\left(\cos ^{-1} \sqrt{\sqrt{13} / 8+1 / 2}, \frac{3-\sqrt{2 \sqrt{13}-5}}{3+\sqrt{2 \sqrt{13}-5}}\right)=(0.2239,0.3372)$.

Equations (13) and (14) together parametrize all the fixed points. They define a surface, or equivalently, a two-parameter family. Sweeping $\beta$ and $\psi$ yields the corresponding $r$ and $A$ values. The resulting surface has two sheets that join along a fold; its projection onto the $(\beta, A)$ plane defines the curve of saddle-node bifurcations.

To calculate the saddle-node curve, we linearize (12) about a fixed point, and set the determinant of the Jacobian to zero. This implies

$$
\sin \beta+\frac{\sin (2 \beta+\psi)[\sin (\beta-2 \psi)+2 \sin (\beta+2 \psi)]}{\sin (2 \beta-\psi)+2 \sin \psi}=0,
$$

where we've used Eqs. (13) and (14) to simplify the determinant. Solving (15) for $\psi(\beta)$ yields two roots, but one of them implies $r>1$ and hence is spurious; the correct root is

$\psi=-2 \beta-2 \beta^{2}+2 \beta^{3}+\frac{11 \beta^{4}}{3}-12 \beta^{5}-\frac{3271 \beta^{6}}{180}+O\left(\beta^{7}\right)$.

This is then substituted into (14) to yield $r(\beta)$, which in turn yields $A(\beta)$, via (13). In this way we obtain the saddle-node curve

$$
A_{S N}(\beta)=2 \beta-2 \beta^{2}-\frac{7 \beta^{3}}{3}+\frac{20 \beta^{4}}{3}+\frac{181 \beta^{5}}{60}+O\left(\beta^{6}\right)
$$

which matches the numerical curve shown in Fig. 4.

To find the Hopf curve, we set the trace of the Jacobian to zero, which gives $\psi=-\frac{1}{2} \sin ^{-1}(2 \sin 2 \beta)$. Repeating the procedure above leads to an exact parametric equa- tion for the Hopf curve. Its leading order behavior is

$$
A_{\mathrm{H}}(\beta)=2-\sqrt{3}+(4 \sqrt{3}-6) \beta^{2}+\left(\frac{26}{\sqrt{3}}-10\right) \beta^{4}+O\left(\beta^{6}\right) .
$$

Future work should investigate whether breathing chimeras exist for the one- and two-dimensional arrays of oscillators studied previously [3, 4, 5, 6, 7]. Are the stability diagrams for such systems similar to Fig. 4? Do chimeras also exist if the oscillators are non-identical [10, 11, 12] or arranged in complex networks [13]? It would also be worth looking for experimental examples of chimera states. Candidate systems include arrays of lasers [14] and chemical [15] or electrochemical [16] oscillators.

We thank E. Ott and T. M. Antonsen for sending us their preprint. Research supported by NSF grant DMS0412757 to S.H.S. and a Mathematical Sciences Postdoctoral Research fellowship to D.M.A.

* Electronic address: strogatz@cornell.edu

[1] N. C. Rattenborg, C. J. Amlaner and S. L. Lima, Neuroscience and Biobehavioral Reviews 24, 817 (2000).

[2] C. G. Mathews, J. A. Lesku, S. L. Lima, and C. J. Amlaner, Ethology 112, 286 (2006).

[3] Y. Kuramoto and D. Battogtokh, Nonlinear Phenom. Complex Syst. 5, 380 (2002).

[4] D. M. Abrams and S. H. Strogatz, PRL 93, 174102 (2004); Int. J. Bif. and Chaos 16, 21 (2006).

[5] S. I. Shima and Y. Kuramoto, PRE 69, 036213 (2004).

[6] O. E. Omel'chenko, Y. L. Maistrenko, and P. A. Tass, PRL 100, 044105 (2008).

[7] G. C. Sethia, A. Sen, and F. M. Atay, PRL 100, 144102 (2008).

[8] H. Sakaguchi, PRE 73, 031907 (2006); Y. Kawamura, PRE 75, 056204 (2007).

[9] R. Hoyle, Pattern Formation (Cambridge U. Press, Cambridge, U. K., 2006).

[10] Y. Kuramoto, Chemical Oscillations, Waves, and Turbulence (Springer, New York, 1984); A. Pikovsky, M. Rosenblum, and J. Kurths, Synchronization (Cambridge U. Press, Cambridge, U. K., 2003); S. Manrubia, A. S. Mikhailov, and D. H. Zanette, Emergence of Dynamical Order (World Scientific, Singapore, 2004).

[11] E. Ott and T. M. Antonsen, arXiv:0806.0004

[12] E. Barreto, B. Hunt, E. Ott, and P. So, arXiv:0706.4454.

[13] M. Barahona and L. M. Pecora, PRL 89, 054101 (2002); S. Boccaletti et al., Phys. Rep. 366, 1 (2002); M. Timme, F. Wolf, and T. Geisel, PRL 92, 074101 (2004); A. E. Motter, C. Zhou and J. Kurths, PRE 71, 016116 (2005); J. G. Restrepo, E. Ott, and B. R. Hunt, Chaos 16, 015107 (2006).

[14] F. Rogister and R. Roy, PRL 98, 104101 (2007).

[15] V. K. Vanag and I. R. Epstein, PRL 87, 228301 (2001).

[16] F. Plenge, H. Varela, and K. Krischer, PRL 94, 198301 (2005). 